\title{
Facial Nerve Intraoperative Monitoring in Otologic Surgeries under Sedation and Local Anesthesia - A Case Series and Literature Review
}

\author{
Lucas Resende Lucinada Mangia1@ Vanessa Mazanek Santos ${ }^{2}$ Thaisa Muniz Mansur ${ }^{1}$ \\ Gislaine Richter Minhoto Wiemes ${ }^{1}$ Rogerio Hamerschmidt ${ }^{1,2}$
} ${ }^{1}$ Department of Otorhinolaryngology - Head and Neck Surgery, Hospital
de Clínicas, Universidade Federal do Paraná, Curitiba, PR, Brazil
2 Otology Center, Instituto Paranaense de Otorrinolaringologia,
Curitiba, PR, Brazil

\begin{abstract}
Address for correspondence Lucas Resende Lucinda Mangia, MD Departamento de Otorrinolaringologia - Cirurgia de Cabeça e Pescoço, Universidade Federal do Paraná, Rua General Carneiro 181, Curitiba, PR, 80060-000, Brazil (e-mail: lucasrlmangia@gmail.com).
\end{abstract}

Int Arch Otorhinolaryngol 2020;24:e11-e17.

\begin{abstract}
Keywords

- facial nerve

- local anesthesia

- otologic surgical procedures

Introduction Local anesthesia with sedation has been employed for an increasingly number of otolaryngology procedures, and might be associated with lower surgical morbidity and costs. Facial nerve monitoring is often advisable in otology to minimize the risks of injuries to this cranial nerve, but the principles, techniques and parameters involved have only been studied for procedures under general anesthesia.

Objective To report the preliminary outcomes of intraoperative facial nerve monitoring during otologic procedures under sedation and local anesthesia.

Methods A total of five procedures and their respective intraoperative electrophysiological main findings were described. Facial neuromonitoring was performed using the same device by an electrophysiologist. The monitor sensitivity was set at $100 \mathrm{mV}$, and a stimulating probe was used whenever needed.

Results Progressively decreasing low-amplitude baseline values were usually obtained as the level of anesthesia increased, with isolated oscillations possibly related to some degree of voluntary muscular activity. These oscillations could be easily distinguished from those of the surgical manipulation or electrical stimulation of the nerve, which tended to be of much greater amplitude and shorter latency, occurring during specific surgical steps.

Conclusion With a surgical team with proper procedural knowledge and broad expertise regarding the technique, intraoperative facial nerve monitoring under local anesthesia with sedation seemed both feasible and reliable. Thus, the need for intraoperative neuromonitoring should not be an obstacle for otologic procedures under less aggressive anesthetic management.
\end{abstract}

\section{Introduction}

Surgical procedures in modern medicine have progressively evolved toward the use of minimally-invasive and more conservative techniques, both as a demand of the patients and as a possibility brought by state-of-the-art technologies and avant-garde studies. These contemporary approaches

received

December 19, 2018

accepted

July 27, 2019
DOI https://doi.org/

10.1055/s-0039-1697991. ISSN 1809-9777. would be considered effective if they incurred in reduced stress response to trauma and lower intraoperative risks without compromising the surgical outcomes. Within this context, the advent of surgical techniques with smaller skin incisions, video-assisted procedures and, more recently, robotic surgery has been observed. Developments in the anesthetic practice would also raise the prospect of
Copyright $(2020$ by Thieme Revinter Publicações Ltda, Rio de Janeiro, Brazil
License terms

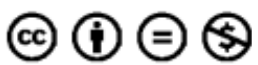


performing surgical procedures with decreased risks and morbidity along with improved comfort for the patients. Hence, integrating safe and high-quality surgical care with low-risk anesthesia in a clinically-applicable fashion should continuously be the ultimate goal of surgical innovations.

In otolaryngology, and more specifically in otology, one of the most feared complications is facial nerve injury, which is associated with various grades of muscle weakness and subsequent impairment in facial expression. This chain of events might lead to significant esthetic and functional consequences. The facial nerve runs a complex route within the temporal bone in such a way that otologic procedures are often performed near the nerve canal, which is known as the Fallopian canal, or use it as a reliable landmark.

The concerns regarding facial nerve injury have led to the emergence and wide use of intraoperative neuromonitoring systems, which have been thoroughly studied and validated for surgeries under general anesthesia. ${ }^{1,2}$ Cochlear implantations, for example, are among the surgeries during which injuries to the seventh cranial nerve are most likely and feared. When performing posterior tympanotomy, the surgical step in which the facial recess is opened and widened to enable the insertion of the electrode array, the nerve lies just underneath the drilling site. Thus, cochlear implant surgeries are regarded as an intervention in which facial nerve monitoring is classically advocated. However, other surgeries such as those for treating chronic otitis also pose a risk to this nerve, as the pathological tissue might surround the Fallopian canal and sometimes the nerve sheath itself.

Local anesthesia with sedation has been successfully employed for an increasingly number of procedures across otolaryngology. Although this anesthetic management was initially established for nasal surgeries, it proved effective in such otologic procedures as tympanoplasty, tympanomastoidectomy, stapedectomy, and cochlear implantation., ${ }^{3,4}$ Nonetheless, the principles, techniques and parameters of use of facial nerve monitoring under local anesthesia with conscious sedation have not yet been studied, determined or reported in the medical literature.

The present study has the goal of reporting the preliminary outcomes of facial nerve intraoperative monitoring (FNIM) during otologic procedures under sedation and local anesthesia to suggest methods and techniques for its safe and effective use.

\section{Methods}

Herein we will describe and discuss the perioperative electrophysiological data obtained from five otologic procedures in four adult patients. These procedures were performed by the same surgeon, with the patients under local anesthesia and sedation. The preoperative imaging assessment included a computed tomography scan for each case and the images did not show any temporal bone malformation in the patients included. Moreover, upon clinical examination, they did not present any limitation in facial muscle tone or expression.
The extensive preoperative anesthetic consultation consisted of evaluation of the patients' clinical condition, investigation of possible coexisting diseases, and a thorough explanation of the anesthetic procedures required intraoperatively.

No premedication was administered. The intraoperative basic monitoring included electrocardiogram and pulse oximetry. Fentanyl $1 \mathrm{ucg} / \mathrm{kg}$, meperidine $0.5 \mathrm{mg} / \mathrm{kg}$, midazolam $5 \mathrm{mg}$, and clonidine $2 \mathrm{ucg} / \mathrm{kg}$ upon induction of the anesthesia were administered to both patients. An oxygen flow of $3 \mathrm{~L} /$ min was delivered by nasal cannula. Local infiltration anesthesia was then performed by the surgeon, using a solution of $2 \%$ lidocaine with 1:50.000 epinephrine. This infiltration was performed at the postauricular area, near the postauricular sulcus, and at the four quadrants of the external auditory canal, as traditionally described for otologic procedures. A total volume of $6-8 \mathrm{~mL}$ of anesthetic solution was injected for each patient. During surgery, opioids would be re-administered if any signs of pain or discomfort were perceived. Other drugs routinely administered were: ondansetron $4 \mathrm{mg}$, metoclopramide $10 \mathrm{mg}$, cefazolin $1 \mathrm{~g}$, dexamethasone $1 \mathrm{mg} / \mathrm{kg}$, dypirone (metamizole) $1 \mathrm{~g}$, and ketorolac $30 \mathrm{mg}$. Reversal of morphine anesthesia, if needed, could be achieved by administering naloxone $0.2 \mathrm{mg}$.

Facial nerve monitoring was performed using the NIMNeuro 3.0 (Medtronic Xomed Inc., Jacksonville, FL, US) nerve integrity monitor by a specialized electrophysiologist. Previously to the local infiltration, but after sedating the patient, intramuscular-type electrodes were inserted to capture the facial muscle responses. Paired electrodes were inserted at least into the frontalis, orbicularis oris and mentalis muscles, along with a ground electrode. In some cases, an additional pair was inserted into the orbicularis oris muscle. During surgery, the electrophysiologist was responsible for evaluating the electrophysiological parameters obtained, that is, the electric signal recorded and originated from the muscle membrane potential.

The monitor's sensitivity was set at $100 \mathrm{mV}$. This means that any source of irritation, regardless of its origin, that led to stimuli of the facial nerve higher than $100 \mathrm{mV}$ would result in typical oscillations around the baseline, with the concomitant emission of a warning noise. If necessary, a specific type of probe could also be used to identify the nerve within the surgical field. For this purpose, the tip of the probe is placed on any structure, and then discharges an electric current whose impact on the baseline register helps to define if the targeted structure corresponds to the nerve itself. Moreover, an analysis of the repercussion of the changes in the amperage of the emitted current on the latency and amplitude of the neural response might be helpful in diagnosing potential lesions suspected perioperatively.

\section{Results}

The five procedures (four patients in total) herein described were uneventful, with satisfactory surgical and anesthetic outcomes. The summary of the data obtained are shown in -Table 1. 
Table 1 Summary of the information regarding the five procedures with intraoperative facial nerve electrophysiological records described

\begin{tabular}{|c|c|c|c|c|c|}
\hline Patient & $\begin{array}{l}\text { Age } \\
\text { (years) }\end{array}$ & Gender & Procedure & $\begin{array}{l}\text { Otologic } \\
\text { background }\end{array}$ & Electrophysiological findings \\
\hline Case 1 & 51 & Female & $\begin{array}{l}\text { Endoscopic cholestea- } \\
\text { toma removal and } \\
\text { tympanoplasty }\end{array}$ & Chronic otitis & $\begin{array}{l}\text { Progressively decreasing low-amplitude } \\
\text { baselines, with irregular initial moderate } \\
\text { oscillations in the mentalis electrode com- } \\
\text { patible with voluntary muscle activity. Major } \\
\text { alterations during surgical manipulation and } \\
\text { probe-induced nerve stimulation. }\end{array}$ \\
\hline Case 2 & 40 & Male & $\begin{array}{l}\text { Left cochlear } \\
\text { implantation }\end{array}$ & $\begin{array}{l}\text { Temporal bone } \\
\text { fracture }\end{array}$ & $\begin{array}{l}\text { Progressively decreasing low-amplitude } \\
\text { baselines. Mild alterations close to the skin } \\
\text { incision. No oscillations detected during } \\
\text { other specific surgical steps. }\end{array}$ \\
\hline Case 3 & 40 & Male & $\begin{array}{l}\text { Right cochlear } \\
\text { implantation }\end{array}$ & $\begin{array}{l}\text { Temporal bone } \\
\text { fracture }\end{array}$ & $\begin{array}{l}\text { Low-amplitude and more stable baselines } \\
\text { since the beginning of the surgery. The use of } \\
\text { the stimulation probe led to a short-latency } \\
\text { and high-amplitude neuromuscular response. }\end{array}$ \\
\hline Case 4 & 68 & Male & $\begin{array}{l}\text { Right cochlear } \\
\text { implantation }\end{array}$ & $\begin{array}{l}\text { Idiopathic sensorineu- } \\
\text { ral hearing loss }\end{array}$ & $\begin{array}{l}\text { Progressively decreasing low-amplitude } \\
\text { baselines. Mild alterations close to the pos- } \\
\text { terior tympanotomy, but without triggering } \\
\text { the alarm. }\end{array}$ \\
\hline Case 5 & 34 & Male & $\begin{array}{l}\text { Left cochlear } \\
\text { implantation }\end{array}$ & Advanced otosclerosis & $\begin{array}{l}\text { Progressively decreasing low-amplitude } \\
\text { baselines, with mild non-significant oscilla- } \\
\text { tions during the use of the drill. Major alter- } \\
\text { ations during voluntary manipulation of an } \\
\text { area of facial nerve dehiscence and the use of } \\
\text { the stimulation probe. }\end{array}$ \\
\hline
\end{tabular}

The first patient, NSCBR, a 51-year-old female with clinical history of controlled hypertension (American Society of Anesthesiologists [ASA] grade II), was submitted to the endoscopic management of a limited attic cholesteatoma with concomitant tympanoplasty. A possible dehiscence of the facial nerve in the tympanic segment was suspected in the preoperative assessment and confirmed intraoperatively. - Fig. 1 summarizes the intraoperative record of the facial neuromuscular electric activity. As the procedure began, with the patient still under mild sedation, low-amplitude $(20-8+0 \mu \mathrm{V})$ baseline values were detected. However, the amplitudes were higher than those habitually observed during facial nerve monitoring upon general anesthesia. The mentalis baseline value showed an irregular pattern, with much greater amplitude, varying from 200 to $300 \mu \mathrm{V}$ (-Fig. 1a). This finding was compatible with the presence of correspondent muscle activity and noisy breathing. To avoid repetitive false alarms, the data provided by this electrode were excluded from analysis in the beginning of the procedure.

As the surgery progressed and the patient achieved a stronger anesthesia effect, there was a perceivable reduction in the overall mean amplitude for all electrodes (5-60 $\mu \mathrm{V}$ for the 3 upper electrodes, $60-90 \mu \mathrm{V}$ for the mentalis electrode) (-Fig. 1b). The use of the drill in the attic/epitympanum led to a mild increase in the mean amplitudes, although it remained inferior to $100 \mu \mathrm{V}$ (-Fig. 1c). Nonetheless, manipulation of the facial nerve sheath in the tympanic segment while debulking squamous debris generated great oscilla- tions on the values for the frontalis and orbicularis oculi electrodes - the latter reaching $143 \mu \mathrm{V}$ and determining the alarm activation and concomitant sound emission (-Fig. 1d). The use of the testing probe in the facial nerve pathway resulted in high-amplitude ( $>700 \mu \mathrm{V})$ and short-latency (4 ms) oscillations (-Fig. 1e).

The second patient (second and third procedures) was a 40-year-old male, JAP, who underwent a simultaneous bilateral cochlear implantation (Nucleus, Cochlear Ltd., Sydney, Australia) to address a bilateral anacusis secondary to head trauma with temporal bone fractures. He had no preoperative comorbidity (ASA I), and, despite the complex bilateral fractures, he showed no paralyzed facial muscles at any moment before the procedure. The surgery began with the left side. A continuous low-amplitude baseline electrical value was initially recorded. As the procedure progressed and the anesthesia intensified, the mean baseline amplitude descended. Isolated major transitory oscillations were observed close to the incision time, with the patient still mildly sedated, which were concomitant and compatible with voluntary movements. No specific alterations could be detected during the detachment of the soft tissues, the bone drilling, and the posterior tympanotomy. As the patient had already been properly sedated, in the beginning of the procedure on the right side, no oscillations in the recordings could be noticed. The baseline amplitude for this procedure remained comparatively lower and more stable. Similarly, there were no significant oscillations during specific surgical steps. Upon the use of the stimulation probe in a presumed 


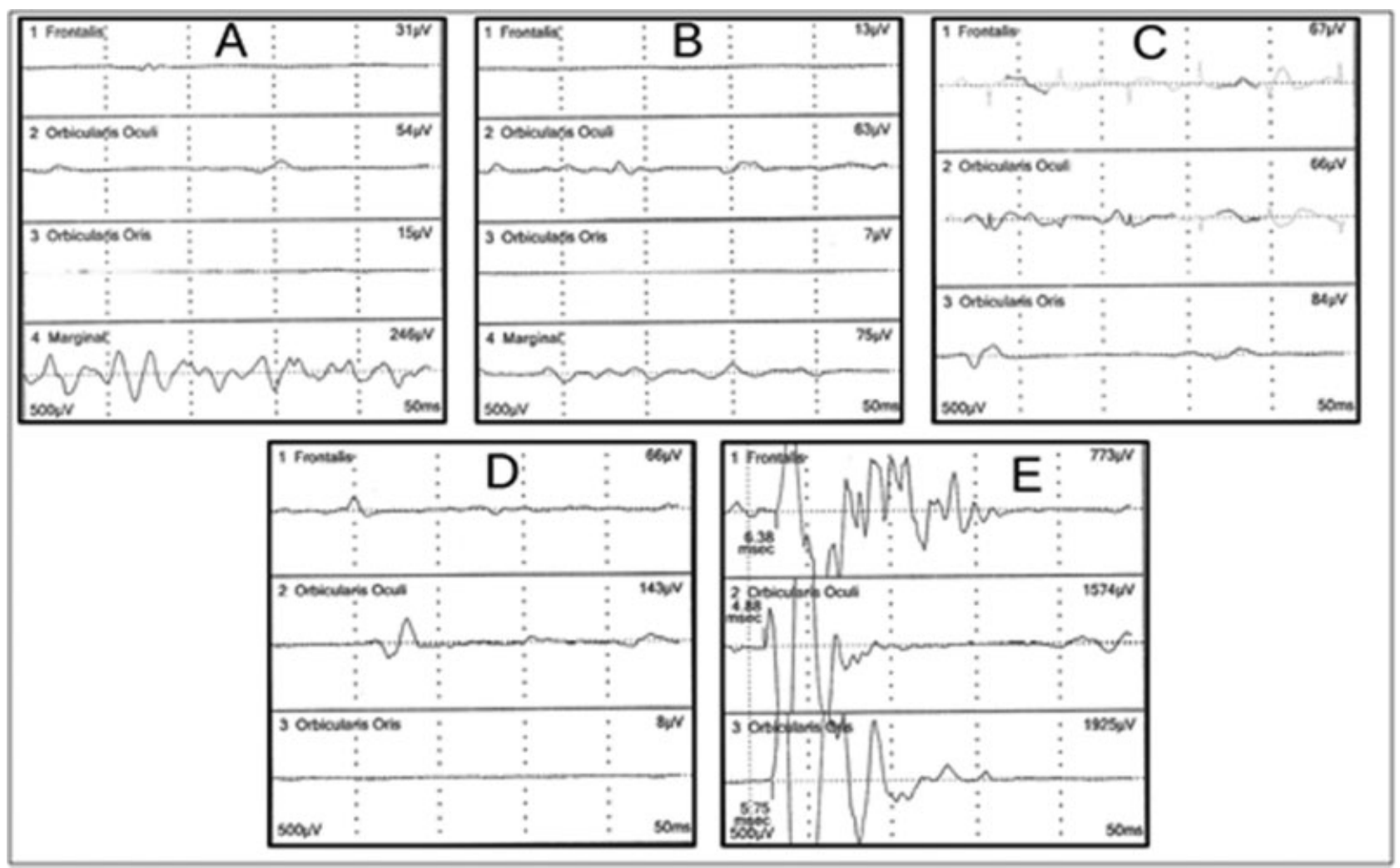

Fig. 1 Electrical records obtained for facial nerve monitoring during different surgical steps of an endoscopic approach to a limited attic cholesteatoma. (A) Beginning of the surgery, with the patient under mild sedation; (B) patient under deeper anesthetic effect; (C) use of the drill in the surgical site; (D) debulking of the cholesteatoma over an area of facial nerve dehiscence; (E) direct stimulation of the facial nerve with the testing probe.

area of the Fallopian canal on the right side, a high-amplitude, short-latency oscillation was observed for all of the electrodes inserted.

The fourth patient, AZ, was a 68 -year-old adult male submitted to a cochlear implant surgery (Nucleus). He had been suffering from profound bilateral progressive hearing loss of unknown cause. He had only mild drug-controlled systemic hypertension (ASA II). In this case, stable, low-amplitude values were recorded throughout the whole procedure. During the manipulation of the nerve behind the posterior tympanotomy, an isolated wave of moderate amplitude arose in record of the orbicularis oris muscle. This timely oscillation enabled a controlled and safe drilling of the facial recess.

The fifth procedure was a unilateral cochlear implant surgery (Nucleus). The patient, AP, a 34-year-old male, developed profound bilateral hearing loss due to advanced otosclerosis. He had no other comorbidities (ASA I). A total of four pairs of electrodes were placed in the same way as described for the first patient. - Fig. 2 summarizes the electrical values during the main surgical events. Initial low-voltage waveforms were obtained for all electrodes, although they were slightly superior for the orbicularis oculi muscle (-Fig. 2a). During skin incision, as the patient was under deeper sedation, the baseline amplitude had noticeably diminished (-Fig. 2b). A mild non-significant increase could be observed during the use of the drill ( - Fig. 2c). The use of the stimulation probe in the facial nerve pathway provoked an immediate neuromuscular response with great amplitude and short latency (-Fig. 2d) - similar to that obtained during the voluntary manipulation of a dehiscent segment of the facial nerve with a Rosen elevator (-Fig. 2e). However, the amplitudes recorded during the mechanical manipulation were distinctly lower than those induced by the electrical stimulus.

\section{Discussion}

Intraoperative neurophysiological monitoring or intraoperative neuromonitoring (IOM) is a well-established tool used during procedures involving the possibility of iatrogenic nerve injuries. The first records of the practical use of this technique date back to 1930 , during epilepsy surgeries. ${ }^{5}$ Nevertheless, the use of IOM only became popular and widespread in the early 1980 s. $^{6}$ Since then, major improvements have been achieved due to the scientific and technological developments in the field of electrophysiology. This surgical tool is nowadays broadly used preventively to lower iatrogenic risks, as it enables the identification of important neural structures in the operative field. ${ }^{7}$ The neural response may be assessed intraoperatively by different methods: motorevoked potentials (MEPs), somatossensory-evoked potentials (SSEPs), electroencephalography (EEG), electromyography (EMG), brainstem auditory-evoked potentials (BAEPs) and visual-evoked potentials (VEPs). 


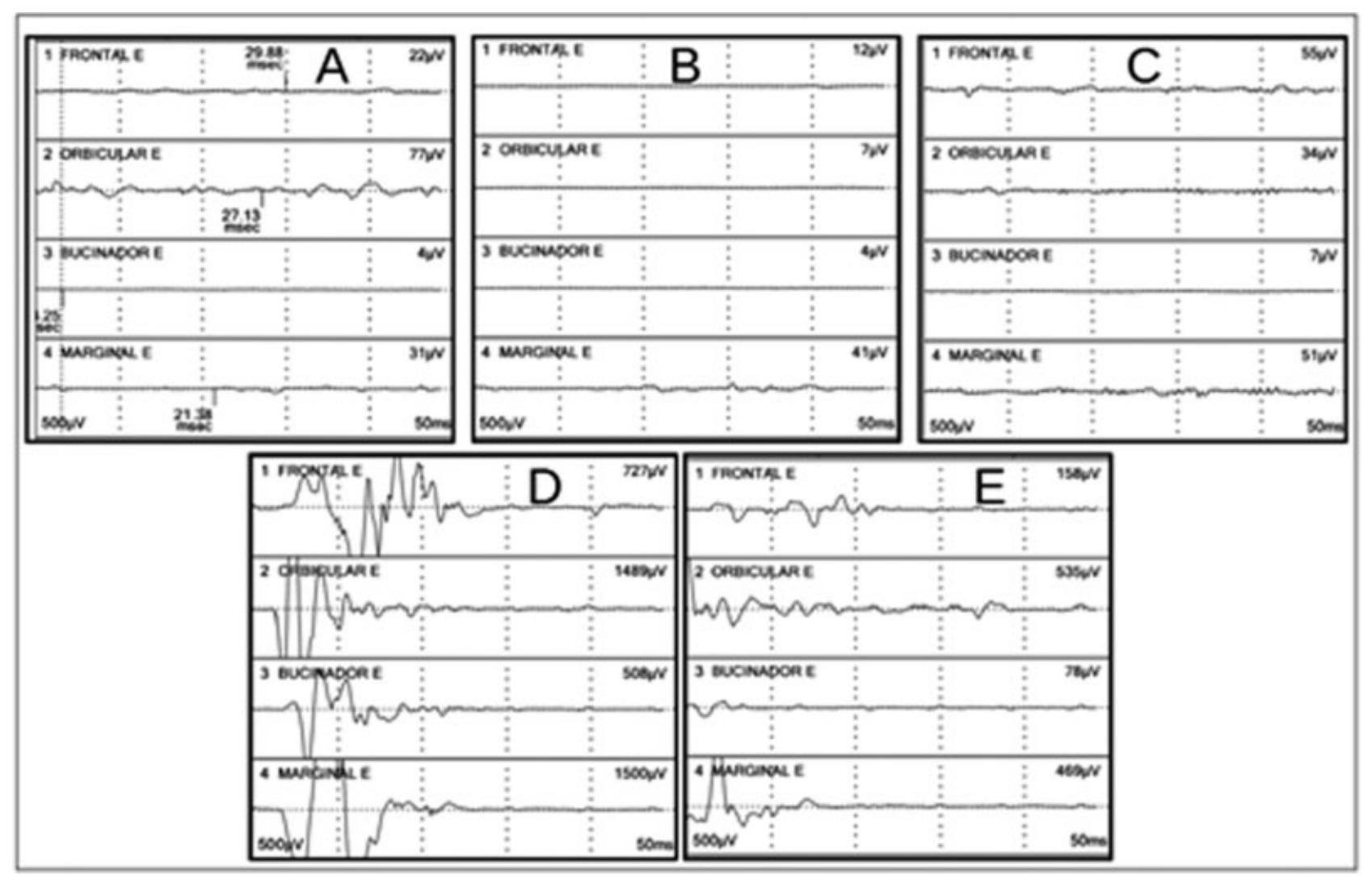

Fig. 2 Electrical records obtained for facial nerve monitoring during different surgical steps of a cochlear implantation. (A) Beginning of the surgery, with the patient under mild anesthesia; (B) patient under deeper anesthesia; (C) use of the drill when performing the posterior tympanotomy; (D) direct stimulation of the facial nerve with the probe; (E) voluntary facial nerve manipulation over an area of dehiscence.

Among these surgical tools, EMG is the most frequently used during otologic procedures, and it consists of recording skeletal muscle activity by measuring and tracking changes in the muscle cell membrane potential. It might be obtained with either intramuscular or surface electrodes. Within this context, the muscular fibers innervated by a single motor axon would form a motor unity, ${ }^{8}$ whose electrical activity would collectively determine the motor unity action potentials. ${ }^{9}$ The motor-evoked responses obtained in the EMG, therefore, mirror the anatomical and physiological properties of the muscle under evaluation. Thus, it might be employed to investigate abnormalities in the skeletal system and determine signs of muscular dysfunction, evaluate central or peripheral neuropathies, estimate the prognosis and severity of neuromuscular disorders, and monitor the efficacy of different therapeutic approaches. ${ }^{10}$

Previous studies have established the accuracy of intraoperative monitoring in facial nerve identification, ${ }^{11}$ as well as the cost-effectiveness when used for ear and temporal bone surgeries. ${ }^{12}$ The indications for facial nerve monitoring have become increasingly diverse, and include parotidectomies, revision tympanoplasties and/or tympanomastoidectomies, cochlear implantations, surgical treatment of middle or posterior fossa tumors, and any other otologic procedure that may pose a risk to the VII cranial nerve. ${ }^{1,13}$ Besides, a study by $\mathrm{Hu}$ et $\mathrm{al}^{14}$ suggests a contemporary trend toward the increased use of nerve monitoring by younger surgeons.
The researchers also found a growing inclination among otologic surgeons towards routine intraoperative monitoring when treating chronic ear diseases. ${ }^{15}$ The main drawback in electromyography-based facial nerve monitoring is the inability to discriminate the proximity of the nerve within the submillimeter range. Although this does not significantly impact the regular otologic procedures performed nowadays, future techniques such as robotic cochlear implantation might need improved automated systems. Previous studies have already shown that an optimized neuromonitoring protocol using an integrating bipolar and monopolar stimulating probe could be safe during the robotic drilling in animal models and for the clinical use. ${ }^{16,17}$ Another preliminary study on this matter has reported that the accuracy of intraoperative tissue discrimination would also increase if impedance spectroscopy was used during robotic mastoid drilling. Therefore, in the future, these techniques may be combined to improve nerve distance assessment in this particular scenario. $^{18}$

The main goals of FNIM are to track the nerve beforehand, to help identify bony canal dehiscence that might increase the surgical risks, and to assess nerve function after the procedure. ${ }^{1,11,19}$ Many factors support FNIM in otology. First, the tridimensional anatomy of the facial nerve within the temporal bone is complex, and the complete delineation of this structure is often hindered by coexisting diseased tissue. Moreover, dehiscent segments are not uncommon, and 
might not be easily told apart from the hypertrophic mucosal lining or granulation tissue. ${ }^{20}$ It should be highlighted that facial paralysis is utterly stigmatizing, and might lead to great functional and esthetic handicaps. Lastly, upon an injury, nerve reconstruction using the techniques currently described does not enable the full restoration of facial mimicry (grades I and II of the House-Brackmann scale, which ranges from I to V). 2,21 This means that even after a successful reconstruction, the best result one could achieve (grade III) would still be consistent with critical remaining sequelae. ${ }^{22}$

A complete FNIM is performed using paired electrodes corresponding to the following muscles: frontalis, orbicularis oculi, orbicularis oris and mentalis. At least three of them are usually used. A ground electrode is also needed, and often placed in the sternum. In the procedures herein described, intramuscular electrodes were preferred. The surgeon also has at his/her disposal a stimulation probe that may be used to intentionally provoke an electrical stimulus on a certain area. With this maneuver, it is possible to investigate the presence of the nerve in the region that is touched. This strategy may work as a navigation system. Whenever an electric current is discharged onto the nerve, an action potential is triggered, leading to facial spasms and specific, short-latency fluctuations in the baseline. Due to this chain of events, the monitor sounds an alarm that alerts the surgeon to facial nerve proximity.

Sustained motor unity potentials with a frequency $>30$ $\mathrm{Hz}$ are called "neurotonic." This type of activity results from intense stimuli, irritation or injury. It appears during FNIM as an unexpected oscillation, whose interpretation depends on the surgical step in which it occurs and on the type of anesthesia to which the patient is submitted. In case of stable general anesthesia, no spontaneous muscular contraction is expected. Thus, in this scenario, any major fluctuation in the baseline records should be thoroughly checked. The stimulation probe may be used to confirm the proximity of the facial nerve to the area that was being manipulated when the abnormal event occurred.

Hence, the characteristics of the electromyographic recordings obtained during FNIM might be significantly affected not only by individual factors, such as obesity and previously existing neuromuscular condition, but also by the anesthetic depth and type. ${ }^{7}$ The most used type of anesthesia when establishing the FNIM parameters is total intravenous anesthesia (TIVA) by combining propofol with opioids. ${ }^{23}$ Although propofol exerts its effects primarily by modulating gamma-aminobutyric acid (GABA) receptors, it is quickly metabolized so that its blood concentration is swiftly lowered to levels in which the neuroelectrophysiological recording is not usually impaired. Greater doses of propofol, however, lead to a significant negative effect in the FNIM. ${ }^{24,25}$ Classically, the administration of neuromuscular blocking agents is thought to be detrimental to intraoperative nerve monitoring, as they act directly in the neuromuscular junction. Choe et $\mathrm{al}^{25}$ have showed, nonetheless, that during TIVA by combing propofol and remifentanil, the controlled use of nondepolarizing neuromuscular blocking agents would not hinder facial nerve monitoring. ${ }^{24}$
Halogenated volatile compounds might completely abolish neuroelectrophysiological responses, and are, therefore, not routinely used when intraoperative nerve monitoring is considered during the surgery. ${ }^{26}$ Many other substances might have a negative impact on nerve monitoring, such as ketamine and muscle relaxants. ${ }^{26}$ None of the drugs used during the procedures described in the present paper (fentanyl, meperidine, midazolam, clonidine and lidocaine, for example) seemed to impair neuromonitoring, as it could be performed without restriction for all of the patients. Therefore, it appears that opioids and benzodiazepines are also compatible with proper intraoperative nerve monitoring.

Temporal bone surgeries in which FNIM is indicated are performed under general anesthesia in most services throughout the world. Some patients, however, might benefit from having their procedures under local anesthesia and sedation. Elderly individuals with preexisting multiple comorbidities, patients with predictable difficult airway management, and individuals with a risk of having arrhythmic events are a few illustrative examples. Besides, general anesthesia incurs in increased hospital stay and costs, slower postoperative recovery and higher anesthetic risks. Among young adults, the major advantages of local anesthesia are the delayed and reduced need for symptomatic therapy and earlier hospital discharge. . $^{3,4}$

When the patients are under sedation, some level of consciousness and spontaneous muscular activity might be present. This might explain isolated oscillations in the intraoperative facial nerve recordings during the surgery. Whenever an electrical event occurs, the surgical team must evaluate the possibility of potential harm to the nerve. This evaluation should include the surgical step in which the event occurred, the corresponding anesthetic depth, the characteristics of the oscillation, and the concomitance of voluntary facial movements. In the beginning of the procedure, before any drilling, for example, the facial nerve is not under major risk, and the patient is not under deep sedation yet. Therefore, minor and temporary oscillations in the electric recording are more frequently observed due to voluntary activity. On the other hand, any alteration appearing during manipulation near the facial nerve canal must serve as an alarm of potential injury, unless there is compelling evidence to the contrary. Tailoring the degree of anesthesia when facing situations of uncertainty might help the team determine the actual neural risk associated with the event. The present study did not aim to compare the objective latency and amplitude levels of facial nerve stimulation under sedation with those under general anesthesia. Nonetheless, within the surgery under sedation and local anesthesia, we could observe that minor interferences and oscillations were not uncommon, although they exhibited different characteristics from those seen during risky nerve manipulation. The latter seems to occur more persistently as the surgical step proceeds and has apparently shorter latencies and greater amplitudes. However, if any uncertainty persists, it should be underlined that the surgeon could also use the stimulation probe to verify the presence of the nerve on the site under manipulation. Hence, in opposition to 
otologic surgeries under general anesthesia, when the patient is under local anesthesia and sedation, minor oscillations in the electrical recording might ensue without any pathological significance. This particularity demands close attention on the part of the surgical team. The present case series preliminarily suggests that FNIM during otologic procedures is also feasible when they are performed under local anesthesia and sedation. It should be emphasized that, in this scenario, any changes in the basal pattern of the EMG activity must be taken into consideration, and that the surgeon's experience and anatomical knowledge are paramount.

There is no other available data in the literature with regard to FNIM during surgeries under local anesthesia and sedation. Nevertheless, the validation of nerve monitoring in this setting is utterly important, as it would increase the safety of the procedures performed under anesthetic techniques with lower morbidity, and reduce the overall costs. Standard facial nerve monitoring systems are suitable even for procedures usually performed under general anesthesia, but that have been already validated under less aggressive anesthetic management, such as cochlear implantations in adults. Thus, the need for intraoperative facial nerve surveillance should not be the factor hindering the choice of type of anesthesia for otologic procedures.

\section{Conclusion}

Intraoperative facial nerve monitoring during otologic procedures under local anesthesia with sedation is feasible and reliable, although it requires a surgical team with proper procedural knowledge and up-to-date technical skills. The interpretation of abnormalities within the recordings in this setting must be timely, considering the surgical anatomy and steps, and the depth of the anesthesia.

\section{Conflict of Interest}

The authors have none to declare.

\section{References}

1 Edwards BM, Kileny PR. Intraoperative neurophysiologic monitoring: indications and techniques for common procedures in otolaryngology-head and neck surgery. Otolaryngol Clin North Am 2005;38(04):631-642, viii

2 Samii M, Gerganov VM, Samii A. Functional outcome after complete surgical removal of giant vestibular schwannomas. J Neurosurg 2010;112(04):860-867

3 Hamerschmidt R, Mocellin M, Gasperin AC, et al. Anestesia local e sedação para cirurgia de implante coclear: uma alternativa possível. Rev Bras Otorrinolaringol (Engl Ed) 2010;76(05):561-564

4 Hamerschmidt R, Moreira AT, Wiemes GR, Tenório SB, Tâmbara EM. Cochlear implant surgery with local anesthesia and sedation: comparison with general anesthesia. Otol Neurotol 2013;34(01): 75-78

5 Penfield W, Boldrey E. Somatic motor and sensory representation in the cerebral cortex of man as studied by electrical stimulation. Brain 1937;60:389-443
6 Nuwer MR. Intraoperative monitoring of neural function. 1st. ed. Amsterdam: Elsevier; 2008

7 Kim SM, Kim SH, Seo DW, Lee KW. Intraoperative neurophysiologic monitoring: basic principles and recent update. J Korean Med Sci 2013;28(09):1261-1269

8 Enoka RM. Morphological features and activation patterns of motor units. JClin Neurophysiol 1995;12(06):538-559

9 Buchtal F. Electromyography in the evaluation of muscle diseases. Meth Clin Neurophysiol. 1991;2:25-45

10 Wu Y, Martínez MÁM, Balaguer PO. Overview of the Application of EMG Recording in the Diagnosis and Approach of Neurological Disorders. In: Turker H, ed. Electrodiagnosis in New Frontiers of Clinical Research. Rijeka: InTech; 2013:1-23

11 Pensak ML, Willging JP, Keith RW. Intraoperative facial nerve monitoring in chronic ear surgery: a resident training experience. Am J Otol 1994;15(01):108-110

12 Noss RS, Lalwani AK, Yingling CD. Facial nerve monitoring in middle ear and mastoid surgery. Laryngoscope 2001;111(05): 831-836

13 Wilson L, Lin E, Lalwani A. Cost-effectiveness of intraoperative facial nerve monitoring in middle ear or mastoid surgery. Laryngoscope 2003;113(10):1736-1745

$14 \mathrm{Hu}$ J, Fleck TR, Xu J, et al. Contemporary changes with the use of facial nerve monitoring in chronic ear surgery. Otolaryngol Head Neck Surg 2014;151(03):473-477

15 Ansó J, Dür C, Gavaghan K, et al. A Neuromonitoring Approach to Facial Nerve Preservation During Image-guided Robotic Cochlear Implantation. Otol Neurotol 2016;37(01):89-98

16 Ansó J, Scheidegger O, Wimmer W, et al. Neuromonitoring During Robotic Cochlear Implantation: Initial Clinical Experience. Ann Biomed Eng 2018;46(10):1568-1581

17 Anso J, Balmer TW, Jegge Y, et al. Electrical Impedance to Assess Facial Nerve Proximity During Robotic Cochlear Implantation. IEEE Trans Biomed Eng 2019;66(01):237-245

18 Kartush JM. Electroneurography and intraoperative facial monitoring in contemporary neurotology. Otolaryngol Head Neck Surg 1989;101(04):496-503

19 Weber PC. Iatrogenic complications from chronic ear surgery. Otolaryngol Clin North Am 2005;38(04):711-722

20 Brackmann DE, Cullen RD, Fisher LM. Facial nerve function after translabyrinthine vestibular schwannoma surgery. Otolaryngol Head Neck Surg 2007;136(05):773-777

21 Schick B, Dlugaiczyk J. Surgery of the ear and the lateral skull base: pitfalls and complications. GMS Curr Top Otorhinolaryngol Head Neck Surg 2013;12:Doc05https://www.egms.de/static/en/ journals/cto/2013-12/cto000097.shtml. AccessedNov122018

22 Liu J, Singh H, White PF. Electroencephalographic bispectral index correlates with intraoperative recall and depth of propofol-induced sedation. Anesth Analg 1997;84(01):185-189

23 Scheufler KM, Zentner J. Total intravenous anesthesia for intraoperative monitoring of the motor pathways: an integral view combining clinical and experimental data. JNeurosurg 2002;96 (03):571-579

24 Sloan TB, Heyer EJ. Anesthesia for intraoperative neurophysiologic monitoring of the spinal cord. J Clin Neurophysiol 2002;19(05): 430-443

25 Choe WJ, Kim JH, Park SY, Kim J. Electromyographic response of facial nerve stimulation under different levels of neuromuscular blockade during middle-ear surgery. J Int Med Res 2013;41(03): 762-770

26 Schubert A, Licina MG, Lineberry PJ. The effect of ketamine on human somatosensory evoked potentials and its modification by nitrous oxide. Anesthesiology 1990;72(01):33-39 